\title{
Berberine protects against diabetic retinopathy by inhibiting cell apoptosis via deactivation of the $\mathrm{NF}-\kappa \mathrm{B}$ signaling pathway
}

\author{
JIAJIA ZHAI ${ }^{1,2^{*}}$, ZEPING LI $^{3 *}$, HUIFENG ZHANG $^{4}$, LOUYAN MA $^{2}$, ZHENGQUAN MA $^{2}$, \\ YI ZHANG ${ }^{5}$, JIAN ZOU $^{6}$, MO LI $^{2}$, LI MA $^{2}$, XIN WANG ${ }^{2}$ and XIAOMIAO LI ${ }^{1}$ \\ ${ }^{1}$ Department of Endocrinology, Xijing Hospital, Air Force Medical University, Xi'an, Shaanxi 710032; \\ ${ }^{2}$ Department of Geratology, Xi'an Ninth Hospital, Xi'an, Shaanxi 710054; ${ }^{3}$ Department of Clinical Medicine, \\ School of Queen Mary, Nanchang University, Nanchang, Jiangxi 330031; ${ }^{4}$ Department of Neurology, Xi'an Electric Power \\ Central Hospital, Xi'an, Shaanxi 710032; ${ }^{5}$ Department of Endocrinology, Xi'an Ninth Hospital, Xi'an, Shaanxi 710054; \\ ${ }^{6}$ Department of Internal Medicine, 522nd Hospital of Chinese People's Liberation Army, Luoyang, Henan 471003, P.R. China
}

Received July 11, 2019; Accepted March 4, 2020

DOI: $10.3892 / \mathrm{mmr} .2020 .11505$

\begin{abstract}
A number of studies have reported that diabetic retinopathy (DR) is the major cause of blindness. Berberine (BBR) is a bioactive constituent that displays effects on blood glucose; however, the mechanism underlying the role of BBR during the development of DR is not completely understood. In the present study, a rat model of DR was successfully established. The eye tissues were removed and subsequently assessed by hematoxylin and eosin staining and the TUNEL assay. The catalase, malondialdehyde, reactive oxygen species, glutathione and superoxide dismutase contents of the eye tissues were measured. Müller cells were chosen for further in vitro experiments. Cell apoptosis was examined by Annexin V-FITC apoptosis detection and Hoechst staining, and the mitochondrial membrane potential was assessed by JC- 1 mitochondrial membrane potential detection. BBR decreased ganglion cell layer, cell apoptosis, reduced diabetic-induced oxidative stress and deactivated the $\mathrm{NF}-\kappa \mathrm{B}$ signaling pathway in the rat model of DR. High glucose enhanced oxidative stress and induced mitochondria-dependent cell apoptosis in Müller cells by activating the $\mathrm{NF}-\kappa \mathrm{B}$ signaling pathway. BBR reversed the high glucose-induced effects by decreasing the phosphorylation of $\mathrm{I} \kappa \mathrm{B}$, inhibiting $\mathrm{NF}-\kappa \mathrm{B}$ nuclear translocation and deactivating the NF- $\mathrm{B}$ signaling pathway. The results suggested that BBR protected against DR by inhibiting oxidative stress and cell apoptosis via deactivation of the $\mathrm{NF}-\kappa \mathrm{B}$ signaling pathway;
\end{abstract}

Correspondence to: Mr. Xiaomiao Li, Department of Endocrinology, Xijing Hospital, Air Force Medical University, 15 West Changle Road, Xi'an, Shaanxi 710032, P.R. China

E-mail: xiaomiao@fmmu.edu.cn

*Contributed equally

Key words: berberine, oxidative stress, cell apoptosis, NF-кB, diabetic retinopathy therefore, suggesting that BBR may serve as a promising therapeutic agent for DR.

\section{Introduction}

Diabetic retinopathy (DR) is a microvascular complication associated with diabetes and is the major cause of blindness $(1,2)$. The prevalence of DR ranges from 15.3 to $42.4 \%$ worldwide, and the risk factors for DR progression include blood glucose, pressure and lipids (3). Although the pathogenesis of DR is complicated, it has been reported that nerve degeneration plays a vital role (4). Müller cells are the primary glial cells in the retina, which provide structural support and the energy required for metabolism. Therefore, Müller cells may have a role in the growth, injury, repair and regeneration of retinal neurons, thus may be used to investigate DR in in vitro studies.

Berberine (BBR) is a bioactive constituent extracted from Rhizoma coptidis that displays effects on congestive heart failure, as well as blood glucose and lipid levels. BBR also protects against oxidative stress-induced injuries (5), including the oxidative stress observed during the early stages of DR development, which is evidenced by increased reactive oxygen species (ROS) (6). Moreover, BBR ameliorates fatty acid- and glutamate-induced oxidative stress, and alleviates oxidative damage-induced apoptosis (7-9). Therefore, BBR might serve as a therapeutic agent for type 2 diabetes mellitus, hyperlipidemia and hypertension (10).

BBR displays a relieving effect on diabetic complications. Glial fibrillary acidic protein, a structural protein, is upregulated in Müller cells in response to retinal injury or stress. Fu et al (11) reported that BBR inhibits modified LDL-induced Müller cell injury by activating the AMPK signaling pathway. In addition, BBR can inhibit leukocyte-mediated killing of vascular endothelium, decrease antioxidant enzyme activities and protect against retinal diseases involving oxidative stress $(12,13)$. The aforementioned effects of BBR suggest that the compound may display therapeutic effects during DR; however, the potential mechanism underlying the effect of BBR in DR is not completely understood. 
The present study investigated the effects of BBR on cell apoptosis and oxidative stress. A rat model of DR was successfully established, and subsequently, blood glucose levels, retinal structures, and the inner and outer nuclear layers were examined in vivo. Müller cells were used to further investigate oxidative stress and cell apoptosis in vitro. The results of the present study may provide a novel insight for the prevention and treatment of DR.

\section{Materials and methods}

Animal experiments. The present study was approved by the Ethics Committee of Xi'an Ninth Hospital and followed the guidelines set by the Association for Research in Vision and Ophthalmology Resolution on Treatment of Animals in Research (14,15). Six-week-old male Sprague-Dawley (SD) rats (weight, $200 \mathrm{~g}$ ) were purchased from Liaoning Changsheng Biotechnology Co., Ltd. All SD rats were housed under standard conditions, with a temperature of $25^{\circ} \mathrm{C}$, humidity of $45-55 \%$, light/dark cycle of $12 / 12 \mathrm{~h}$, and food and water was freely available. Following adaptive feeding for one week, the rats were administered $65 \mathrm{mg} / \mathrm{kg}$ streptozotocin (STZ; cat. no. 18883-66-4; Aladdin; prepared in citrate buffer) to establish the rat model of DR. Control rats were administered $65 \mathrm{mg} / \mathrm{kg}$ citrate buffer. After 3 days, blood glucose levels in blood samples obtained from the tail vein were measured and a glucose level $\geq 300 \mathrm{mg} / \mathrm{dl}$ was considered as a successful establishment of the rat model of DR. BBR was prepared in $0.5 \%$ carboxymethylcellulose (CMC)-Na. A total of $18 \mathrm{DR}$ rats were divided into three groups ( $n=6$ per group): i) The DR group, ii) the DR + BBR-L group and iii) the DR + BBR-H group. The DR group was treated with CMC-Na by gavage for 8 weeks. The DR + BBR-L group was treated with $100 \mathrm{mg} / \mathrm{kg}$ BBR by gavage every day for 8 weeks. The DR + BBR-H group was treated with $200 \mathrm{mg} / \mathrm{kg}$ BBR by gavage every day for 8 weeks.

Body weight and blood glucose levels were measured every 2 weeks for the 8-week treatment period. After an 8-week treatment, the rats were sacrificed by excessive anesthesia with $200 \mathrm{mg} / \mathrm{kg}$ pentobarbital sodium. Death was verified by monitoring cessation of breathing and heartbeats. Subsequently, the eyes were isolated and fixed with $4 \%$ paraformaldehyde for $48 \mathrm{~h}$ at room temperature.

Cell culture. Müller cells are primary glial retina cells and are considered to be the perfect in vitro cell model of DR (16). Müller cells (cat. no. CP-M117; Procell Life Science \& Technology Co., Ltd.) were cultured in Müller cell complete medium (MCCM; cat. no. CM-M117; Procell Life Science \& Technology Co., Ltd.) at $37^{\circ} \mathrm{C}$ with $5 \% \mathrm{CO}_{2}$. After $24-\mathrm{h}$ incubation, Müller cells were incubated for $48 \mathrm{~h}$ with the following: MCCM containing $5.5 \mathrm{mM}$ glucose (control); MCCM containing $33.3 \mathrm{mM}$ glucose [high glucose (HG)]; MCCM containing $33.3 \mathrm{mM}$ glucose and $20 \mu \mathrm{M}$ BBR (HG + $\mathrm{BBR}$ ); and MCCM containing $33.3 \mathrm{mM}$ glucose and $100 \mu \mathrm{M}$ pyrrolidine dithiocarbamate (PDTC, an NF- $\kappa \mathrm{B}$ inhibitor; cat. no. HY-18738; MedChemExpress; HG + PDTC). Subsequently, the treated Müller cells were used for further experiments.

Metabolic analysis. The eye tissues were added to normal saline (weight:volume=1:9) and homogenized. Total protein was quantified using a Bicinchoninic Acid Protein assay kit (cat. no. P0009; Beyotime Institute of Biotechnology), according to the manufacturer's protocols. Subsequently, the catalase (CAT) and superoxide dismutase (SOD) activity, as well as the malonaldehyde (MDA), reactive oxygen species (ROS) and reduced glutathione (GSH) contents of the tissue samples containing equal amounts of protein or treated Müller cells were assessed using the CAT (cat. no. A007-1; Nanjing Jiancheng Bioengineering Institute), MDA (cat. no. A003-1; Nanjing Jiancheng Bioengineering Institute), ROS (cat. no. E004; Jiancheng Bioengineering Institute), SOD (cat. no. A001-1; Jiancheng Bioengineering Institute) and GSH (cat. no. A006-2; Jiancheng Bioengineering Institute) assay kits according to the manufacturer's protocol. The samples were detected using a NovoCyte flow cytometer (ACEA Biosciences, Inc.) and analyzed by NovoExpress ${ }^{\circledR}$ 1.2.5 (ACEA Biosciences, Inc.).

Mitochondrial membrane potential $(\Delta \Psi m)$ assay. Loss of membrane potential $(\Delta \Psi \mathrm{m})$ is a hallmark of cell apoptosis. Following treatment, Müller cells at a density of $90 \%$ were washed with PBS. Subsequently, the mitochondrial membrane potential was measured using a JC-1 mitochondrial membrane potential detection kit (cat. no. C2006; Beyotime Institute of Biotechnology), according to the manufacturer's protocols. The mitochondrial membrane potential was measured using flow cytometry (NovoCyte; ACEA Biosciences, Inc.) and analyzed by NovoExpress 1.2.5 (ACEA Biosciences, Inc.).

Cell Counting Kit-8 (CCK-8) assay. Müller cells were seeded $\left(2 \times 10^{3}\right.$ cells/well) in 96-well plates and incubated for $48 \mathrm{~h}$ at $37^{\circ} \mathrm{C}$ with nine different mediums: i) MCCM (control); ii) $\mathrm{MCCM}$ containing $33.3 \mathrm{mM}$ glucose (HG); iii) MCCM containing $33.3 \mathrm{mM}$ glucose and $1 \mu \mathrm{M}$ BBR $(\mathrm{HG}+1 \mu \mathrm{M}$ BBR); iv) MCCM containing $33.3 \mathrm{mM}$ glucose and $2 \mu \mathrm{M}$ BBR ( $\mathrm{HG}+2 \mu \mathrm{M}$ BBR); v) MCCM containing $33.3 \mathrm{mM}$ glucose and $5 \mu \mathrm{M}$ BBR $(\mathrm{HG}+5 \mu \mathrm{M} \mathrm{BBR})$; vi) MCCM containing $33.3 \mathrm{mM}$ glucose and $10 \mu \mathrm{M} \mathrm{BBR}(\mathrm{HG}+10 \mu \mathrm{M} \mathrm{BBR})$; vii) MCCM containing $33.3 \mathrm{mM}$ glucose and $20 \mu \mathrm{M}$ BBR (HG $+20 \mu \mathrm{M}$ BBR); viii) MCCM containing $33.3 \mathrm{mM}$ glucose and $50 \mu \mathrm{M}$ BBR $(\mathrm{HG}+50 \mu \mathrm{M}$ BBR$)$. Cell viability was detected by CCK-8 assay kit (cat. no. 96992; Sigma-Aldrich; Merck $\mathrm{KGaA}$ ), according to the manufacturer's protocols. Briefly, $100 \mu \mathrm{l}$ MCCM and $10 \mu \mathrm{lCCK}-8$ reagent (Beyotime Institute of Biotechnology) was added to each well, and incubated for $1 \mathrm{~h}$ at $37^{\circ} \mathrm{C}$. The absorbance of each well was recorded at a wavelength of $450 \mathrm{~nm}$ using a microplate reader to identify the optimal therapeutic concentration of BBR.

Western blotting. Total protein from treated Müller cells and eye samples was mechanically lysed using RIPA buffer (cat. no. P0013B; Beyotime Institute of Biotechnology) supplemented with a cocktail of PMSF (cat. no. ST506; Beyotime Institute of Biotechnology). Total protein was extracted using the Nuclear and Cytoplasmic Protein Extraction kit (cat. no. P0028; Beyotime Institute of Biotechnology) or the Mitochondrial Protein Extraction kit (cat. no. AR0156; Boster Biological Technology), according to the manufacturer's protocols. Protein content was measured by the BCA Protein Assay kit (cat. no. P0009; Beyotime Institute of Biotechnology), 
according to the manufacturer's instruction. Proteins (30 $\mu \mathrm{g}$ per lane) were separated by $10 \%$ SDS-PAGE and transferred onto PVDF membranes (cat. no. LC2005; Thermo Fisher Scientific, Inc.). After washing with TBS with $0.15 \%(\mathrm{v} / \mathrm{v})$ Tween-20, the membranes were blocked with $5 \%(\mathrm{~m} / \mathrm{v})$ bull serum albumin (cat. no. BS043; Biosharp Life Sciences) for $1 \mathrm{~h}$ at room temperature and incubated overnight at $4^{\circ} \mathrm{C}$ with primary antibodies targeted against: NF- $\kappa \mathrm{B}$ inhibitor $(\mathrm{I} \kappa \mathrm{B} \alpha$; cat. no. 9242; 1:500; Cell Signaling Technology, Inc.), phosphorylated (p)-IкB $\alpha$ (cat. no. 2859; 1:1,000; Cell Signaling Technology, Inc.), NF-кB p65 (cat. no. 8242; 1:500; Cell Signaling Technology, Inc.), cleaved caspase-3 (cat. no. 9654; 1:500; Cell Signaling Technology, Inc.), cleaved caspase-9 (cat. no. 9507; 1:1,000; Cell Signaling Technology, Inc.), Bcl-2 (cat. no. 12789-1-AP; 1:500; Proteintech), Bax (cat. no. 50599-2-Ig; 1:500; Proteintech Group, Inc.), Cytochrome c (cat. no. 4272; 1:1,000; Cell Signaling Technology, Inc.), COX IV (cat. no. 11242-1-AP; 1:500; Proteintech Group, Inc.), Histone H3 (cat. no. 17168-AP; 1:500; Proteintech Group, Inc.) and $\beta$-actin (cat. no. 60008-1-Ig; 1:2,000; Proteintech Group, Inc.). Subsequently, the membranes were incubated with HRP-conjugated goat anti-rabbit (cat. no. SA00001-2; 1:10,000; Proteintech Group, Inc.) and HRP-conjugated goat anti-mouse (cat. no. SA00001-1; 1:10,000; Proteintech Group, Inc.) secondary antibodies at room temperature for $40 \mathrm{~min}$. Protein bands were visualized using an ECL kit (cat. no. E003; 7Sea Biotech), according to the manufacturer's protocols, and quantified using Gel-Pro-Analyzer software (version 4; Media Cybernetics, Inc.).

TUNEL assay. Cell apoptosis in eye tissues was determined using a TUNEL assay with the In Situ Cell Death Detection kit (cat. no. 11684817910; Merck KGaA), according to the manufacturer's protocols. Eye tissues were cut into $5 \mu \mathrm{m}$ thick slices, dewaxed, dehydrated and washed with PBS. The sections were permeabilized using $0.1 \%$ Triton $\mathrm{X}-100$ for $8 \mathrm{~min}$ at room temperature (cat. no. ST795; Beyotime Institute of Biotechnology), washed with PBS and incubated with TdT Labeling Buffer for $1 \mathrm{~h}$ at $37^{\circ} \mathrm{C}$. Subsequently, the sections were incubated with $50 \mu 1$ Converter-POD (horseradish peroxidase-labeled fluorescein antibody), washed with PBS and developed with DAB (cat. no. DA1010; Beijing Solarbio Science \& Technology Co., Ltd.). After mounting with neutral balsam, TUNEL-positive cells were observed and counted using a fluorescence microscope (magnification, x600) in three random fields.

Hematoxylin \& Eosin $(H \& E)$ staining. SD rats were sacrificed and the eye tissues were isolated. Following dewaxing and dehydration, tissues $(5 \mu \mathrm{m})$ were stained with hematoxylin for $5 \mathrm{~min}$ at room temperature. Then, the eye sections were differentiated using 1\% hydrochloric acid alcohol for $3 \mathrm{sec}$ at room temperature. Subsequently, the sections were washed with $\mathrm{dd}_{2} \mathrm{O}$, stained with eosin for $3 \mathrm{~min}$ at room temperature and dehydrated using an ascending alcohol series for $2 \mathrm{~min}$. The sections were hyalinized using dimethylbenzene, sealed and observed using a light microscope (magnification, x200).

Immunofluorescence staining. Müller Cells were fixed with $4 \%$ paraformaldehyde for $15 \mathrm{~min}$ at room temperature, permeabilized with $0.1 \%$ Triton X-100 (cat. no. ST795; Beyotime Institute of Biotechnology) for $30 \mathrm{~min}$ at room temperature, and washed three times with PBS. Subsequently, the cells were embedded in 5\% blocking serum (cat. no. SL038; Beijing Solarbio Science \& Technology Co., Ltd.) for $15 \mathrm{~min}$ at room temperature. Cells were incubated overnight at $4^{\circ} \mathrm{C}$ with an anti-NF-кB p65 primary antibody (cat. no. \#8242; 1:200; Cell Signaling Technology, Inc.) and subsequently washed with PBS. Following primary incubation, cells were incubated with an FITC-labeled goat anti-rabbit IgG $(\mathrm{H}+\mathrm{L})$ secondary antibody (cat. no. A0562; 1:200; Beyotime Institute of Biotechnology) at room temperature for $60 \mathrm{~min}$. Subsequently, cells were stained with DAPI for $5 \mathrm{~min}$ at room temperature and observed using a DP73 fluorescence microscope (Olympus Corporation; magnification, $\mathrm{x} 400$ ).

Hoechst staining. Following treatment, Müller cells were washed twice with PBS and subsequently stained using the Hoechst Staining kit (cat. no. C0003; Beyotime Institute of Biotechnology), according to the manufacturer's protocols. Stained cells were observed using a DP73 fluorescence microscope (Olympus Corporation; magnification, $\mathrm{x} 400$ ) in three random fields.

Annexin V-FITC apoptosis detection. Apoptotic cells were determined using the Annexin V-FITC Apoptosis Detection kit (cat. no. C1062; Beyotime Institute of Biotechnology), according to the manufacturer's protocols. Briefly, Müller cells of each group at a density of $90 \%$ were seeded into a 6 -well plate and incubated for $48 \mathrm{~h}$ at $37^{\circ} \mathrm{C}$. Subsequently, cells were collected, washed twice with PBS, and stained with $5 \mu 1$ Annexin V-FITC and $10 \mu \mathrm{l}$ propidium iodide for $15 \mathrm{~min}$ at room temperature in the dark. Early apoptotic and necrotic cells were identified using flow cytometry (NovoCyte; ACEA Biosciences, Inc.) and analyzed by NovoExpress 1.2.5 (ACEA Biosciences, Inc.).

Statistical analysis. All experiments were repeated at least three times. Data are presented as the mean \pm SD. Statistical analyses were performed using GraphPad Prism software (version 7.0; GraphPad Software, Inc.). Data containing 2 groups were analyzed using the Student's t-test. Data containing $>2$ groups were analyzed using one-way ANOVA followed by Bonferroni's post hoc test. $\mathrm{P}<0.05$ was considered to indicate a statistically significant difference.

\section{Results}

$B B R$ inhibits retinal ganglion cell apoptosis in a rat model of $D R$. In the present study, a rat model of DR was successfully established by STZ injection. STZ was used to establish a rat model of DR, characterized by high blood glucose levels. Firstly, alterations in body weight and blood glucose levels in response to STZ and BBR were monitored. Body weight (Table I) and blood glucose levels (Table II) were significantly increased in the DR group compared with the control group. Body weight and blood glucose levels were not significantly altered during the 8-week treatment period across the four groups. H\&E (Fig. 1A) and TUNEL (Fig. 1B) staining assays suggested that retinal ganglion cell layer (GCL) apoptosis was 
Table I. Body weight changes (g) in the groups of rats.

\begin{tabular}{llllll}
\hline Group & \multicolumn{1}{c}{0 weeks } & \multicolumn{1}{c}{2 weeks } & \multicolumn{1}{c}{4 weeks } & 6 weeks & 8 weeks \\
\hline Control & $239.00 \pm 11.00$ & $265.50 \pm 14.32$ & $277.83 \pm 15.03$ & $304.67 \pm 30.53$ & $321.33 \pm 33.42$ \\
DR & $240.17 \pm 10.11^{\text {a }}$ & $218.00 \pm 18.35^{\text {a }}$ & $208.33 \pm 18.27^{\mathrm{a}}$ & $194.67 \pm 17.65^{\text {a }}$ & $180.50 \pm 15.08^{\mathrm{a}}$ \\
DR + BBR-L & $237.83 \pm 9.00$ & $217.50 \pm 15.60$ & $204.83 \pm 15.22$ & $188.50 \pm 10.37$ & $173.83 \pm 16.12$ \\
DR + BBR-H & $239.33 \pm 10.88$ & $222.33 \pm 19.01$ & $207.33 \pm 17.84$ & $192.00 \pm 17.37$ & $178.17 \pm 20.00$
\end{tabular}

${ }^{\mathrm{a}} \mathrm{P}<0.0001$ vs. control. DR, diabetic retinopathy; BBR, berberine; BBR-L, $100 \mathrm{mg} / \mathrm{kg}$ BBR; BBR-H, $200 \mathrm{mg} / \mathrm{kg}$ BBR.

Table II. Blood glucose level ( $\mathrm{mg} / \mathrm{dl})$ changes in the groups of rats.

\begin{tabular}{lccccc}
\hline Group & 0 weeks & 2 weeks & 4 weeks & 6 weeks & 8 weeks \\
\hline Control & $5.45 \pm 0.43$ & $5.72 \pm 0.72$ & $5.75 \pm 0.33$ & $5.73 \pm 0.47$ & $5.65 \pm 0.33$ \\
DR & $18.82 \pm 1.45^{\mathrm{a}}$ & $20.37 \pm 1.60^{\mathrm{a}}$ & $21.62 \pm 1.51^{\mathrm{a}}$ & $20.57 \pm 1.93^{\mathrm{a}}$ & $20.42 \pm 1.41^{\mathrm{a}}$ \\
DR+BBR-L & $18.75 \pm 0.89$ & $20.07 \pm 1.45$ & $20.32 \pm 1.28$ & $20.73 \pm 1.67$ & $20.78 \pm 1.60$ \\
DR+BBR-H & $18.88 \pm 1.12$ & $20.07 \pm 1.35$ & $21.18 \pm 1.86$ & $20.68 \pm 0.92$ & $21.33 \pm 1.10$ \\
\hline
\end{tabular}

${ }^{\text {a }}<<0.0001$ vs. control. DR, diabetic retinopathy; BBR, berberine; BBR-L, $100 \mathrm{mg} / \mathrm{kg} \mathrm{BBR}$; BBR-H, 200 mg/kg BBR.

decreased by BBR treatment in the rat model of DR, especially in the BBR-H treatment group. Retinal MDA, SOD, CAT, GSH and ROS contents were measured as metabolic indicators of the eyes (Fig. 1C-G). MDA and ROS contents were significantly increased in the DR group compared with the control group; however, the BBR treated groups displayed significantly decreased MDA and ROS contents compared with the DR group (Fig. 1C and G). Furthermore, GSH levels and antioxidant enzyme activities were significantly decreased in the DR group compared with the control group. The BBR treated groups displayed significantly increased GSH levels and antioxidant enzyme activities compared with the DR group (Fig. 1D-F). The expression levels of p-IкB (Ser32), IкB,

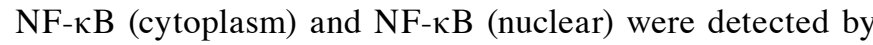
western blotting. The expression levels of $\mathrm{p}-\mathrm{I} \kappa \mathrm{B}$ (Ser32) and $\mathrm{NF}-\kappa \mathrm{B}$ (nuclear) were significantly increased in the DR group compared with the control group, and the BBR treated groups displayed significantly decreased expression levels compared with the DR group (Fig. 1H). However, the expression levels

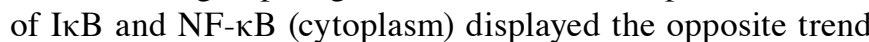
(Fig. 1H). The results suggested that BBR decreased the phosphorylation of I $\kappa$ B at Ser32 and deactivated the NF- $\kappa$ B signaling pathway in the rat model of DR.

Effect of BBR on the viability of Müller cells. Müller cells are primary retinal glial cells. The viability of Müller cells was significantly decreased in the HG group compared with the control group (Fig. 2). To evaluate the effect of BBR on the viability of HG-treated Müller cells, HG-treated Müller cells were incubated with different doses of $\operatorname{BBR}(1,2,5$, 10,20 and $50 \mu \mathrm{M}$ ). BBR treatment increased the viability of HG-treated Müller cells, especially at a concentration of $20 \mu \mathrm{M}$ (P<0.0001; Fig. 2); therefore, $20 \mu \mathrm{M}$ BBR was used for subsequent experiments.
BBR decreases $H G$-induced cell apoptosis and oxidative stress in Müller cells. To investigate the effect of BBR on DR in vitro, HG-treated Müller cells were used to model retinal ganglion cells. Rat models of DR display excessive activation of the NF- $\kappa \mathrm{B}$ signaling pathway; therefore, HG-treated Müller cells were incubated with PDTC (a NF- $\kappa B$ inhibitor) or BBR. Apoptotic cells were identified using Annexin V-FITC apoptosis detection. The rate of apoptosis was significantly increased in the HG group compared with the control group; however, BBR or PDTC treated cells displayed significantly decreased rates of apoptosis compared with the HG group (Fig. 3A). Similar results were also obtained by the Hoechst staining assay (Fig. 3B). Subsequently, apoptosis-related proteins were examined by western blotting. Cleaved caspase-3, cleaved caspase-9 and Bax expression levels were significantly increased in the HG group compared with the control group. HG-treated cells incubated with BBR or PDTC displayed significantly decreased expression levels of cleaved caspase-3, caspase-9 and Bax compared with the HG group (Fig. 3C). The expression of Bcl-2 displayed the opposite trend (Fig. 3C). MMP may be decreased during cell apoptosis; therefore, MMP was evaluated using the JC-1 assay. The results suggested that MMP was decreased in HG-induced Müller cells compared with the control group, and recovered to control levels following treatment with BBR or PDTC (Fig. 3D). The expression of cytoplasmic cytochrome $\mathrm{c}$ was increased and the expression of mitochondrial cytochrome $\mathrm{c}$ was decreased in the HG group compared with the control group. BBR or PDTC treatment reversed HG-induced effects on cytochrome c expression (Fig. 3E). The results suggested that cytochrome c was released from the mitochondria into the cytoplasm following treatment with $\mathrm{HG}$, and BBR or PDTC treatment could reverse HG-induced effects. Furthermore, GSH levels and antioxidant enzyme activity were decreased in the HG 
A

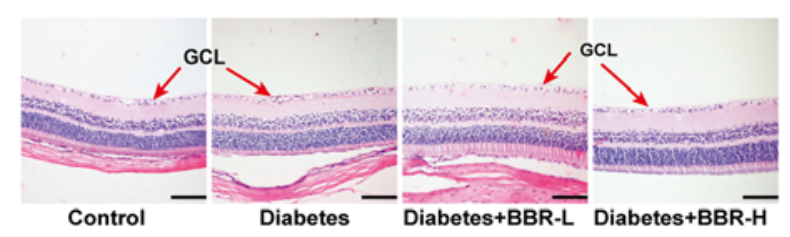

B
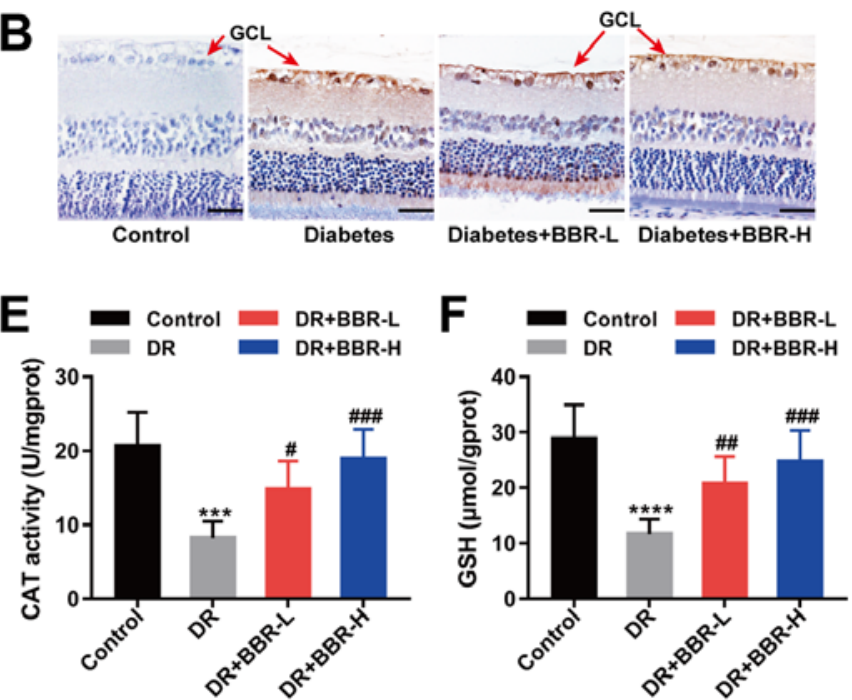

G

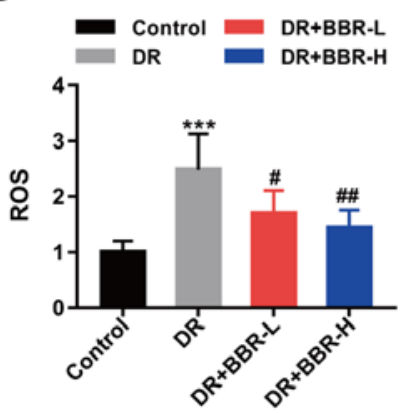

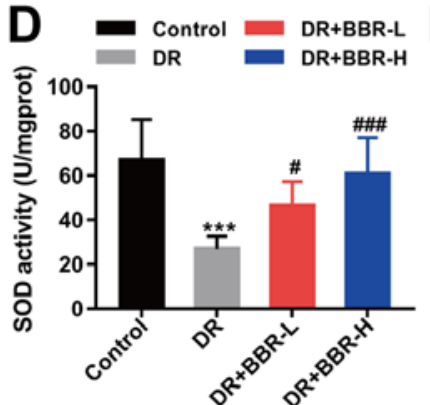

H

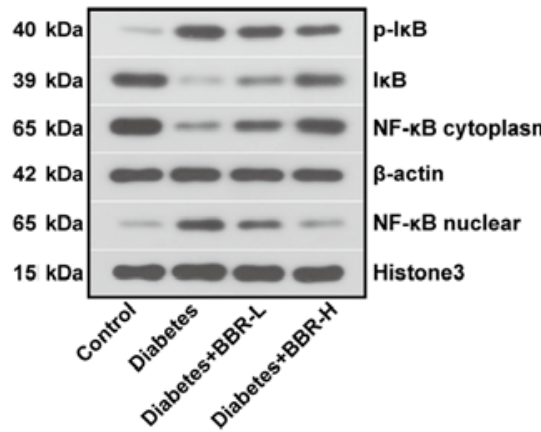

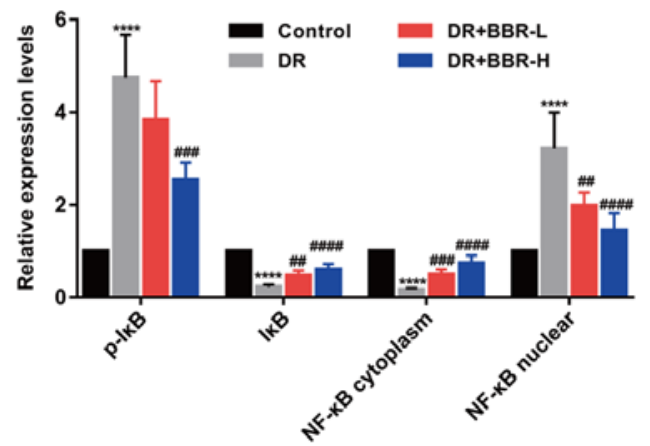

Figure 1. BBR decreases retinal ganglion cell apoptosis in a rat model of DR. (A) Hematoxylin and eosin-stained eye sections (scale bar, $100 \mu \mathrm{m}$ ). (B) Eye sections assessed using the TUNEL staining assay (scale bar, $33 \mu \mathrm{m}$ ). The (C) MDA, (D) SOD, (E) CAT, (F) GSH and (G) ROS contents of the eye sections.

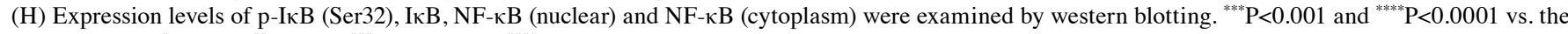
control group. ${ }^{\#} \mathrm{P}<0.05,{ }^{\# \#} \mathrm{P}<0.01,{ }^{\# \# \#} \mathrm{P}<0.001$ and ${ }^{\# \# \# "} \mathrm{P}<0.0001$ vs. the DR group. BBR, berberine; DR, diabetic retinopathy; MDA, malondialdehyde; SOD,

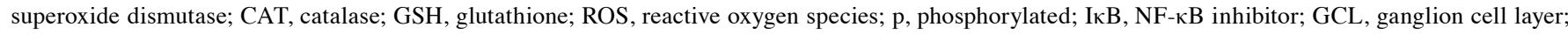
BBR-L, $100 \mathrm{mg} / \mathrm{kg}$ BBR; BBR-H, $200 \mathrm{mg} / \mathrm{kg}$ BBR.

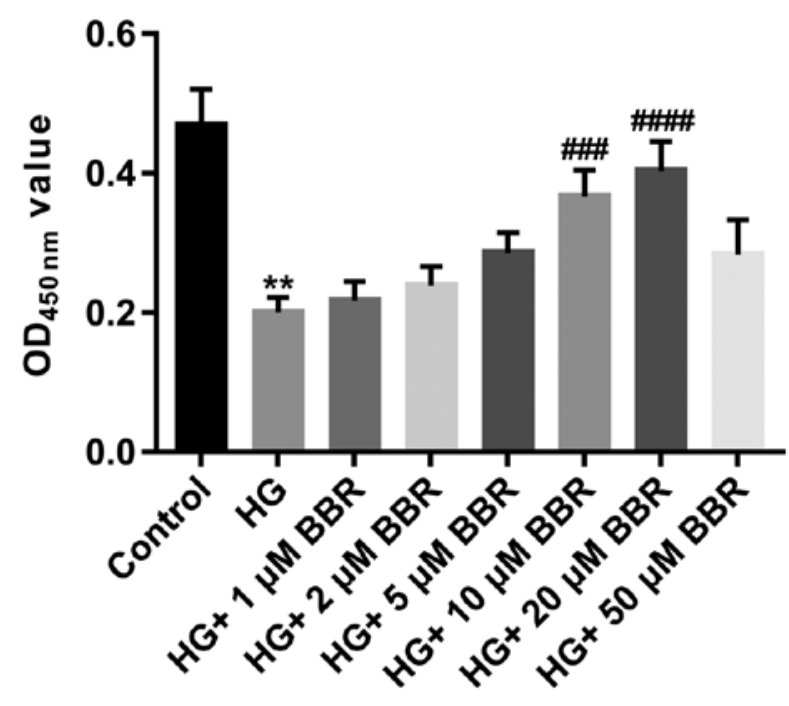

Figure 2. Effect of BBR on Müller cell viability. Müller cells were treated with $5.5 \mathrm{mM}$ glucose; $33.3 \mathrm{mM}$ glucose; $33.3 \mathrm{mM}$ glucose and $1 \mu \mathrm{M} \mathrm{BBR}$; $33.3 \mathrm{mM}$ glucose and $2 \mu \mathrm{M} \mathrm{BBR} ; 33.3 \mathrm{mM}$ glucose and $5 \mu \mathrm{M}$ BBR; $33.3 \mathrm{mM}$ glucose and $10 \mu \mathrm{M}$ BBR; $33.3 \mathrm{mM}$ glucose and $20 \mu \mathrm{M}$ BBR; or $33.3 \mathrm{mM}$ glucose and $50 \mu \mathrm{M}$ BBR. Cell viability was assessed using the Cell Counting Kit-8 assay. ${ }^{* *} \mathrm{P}<0.01$ vs. the control group. ${ }^{\# \# \#} \mathrm{P}<0.001$ and ${ }^{\# \# \# \#} \mathrm{P}<0.0001$ vs. the HG group. BBR, berberine; HG, high glucose; OD, optical density. group compared with the control group, but increased in the BBR and PDTC treated groups compared with the HG group. MDA content displayed the opposite trend (Fig. 3F). ROS levels were detected by flow cytometry, which suggested that the HG group displayed increased ROS levels compared with the control group, but BBR or PDTC treated groups showed decreased ROS levels compared with the HG group (Fig. 3G). The results suggested that BBR reversed HG-induced effects on cell apoptosis and oxidative stress in Müller cells.

$B B R$ decreases cell apoptosis and oxidative stress by deactivating the $N F-\kappa B$ signaling pathway. To investigate the association between $\mathrm{NF}-\kappa \mathrm{B}$ and BBR, the expression

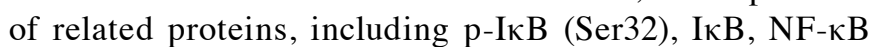
(nuclear), NF- $\kappa \mathrm{B}$ (cytoplasm), were assessed by western blotting (Fig. 4A). The expression levels of p-IкB (Ser32) and $\mathrm{NF}-\kappa \mathrm{B}$ (nuclear) were significantly upregulated in HG-induced Müller cells compared with control cells. However, BBR or PDTC treated Müller cells showed significantly decreased expression levels of $\mathrm{p}-\mathrm{I} \kappa \mathrm{B}$ (Ser32) and NF- $\kappa \mathrm{B}$ (nuclear) compared with the HG group. I $\kappa \mathrm{B}$ and NF- $\kappa \mathrm{B}$ (cytoplasm) expression displayed the opposite trend (Fig. 4A). NF- $\kappa \mathrm{B}$ translocation was determined using an immunofluorescence 
A

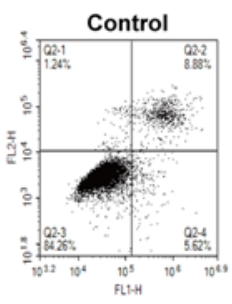

B

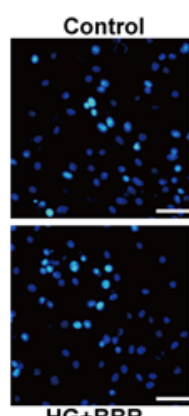

HG+BBR
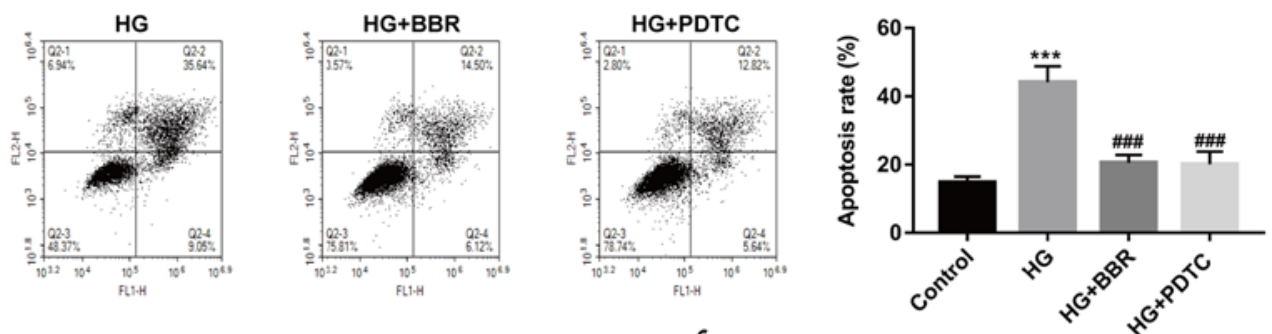

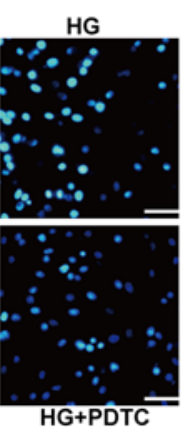

C
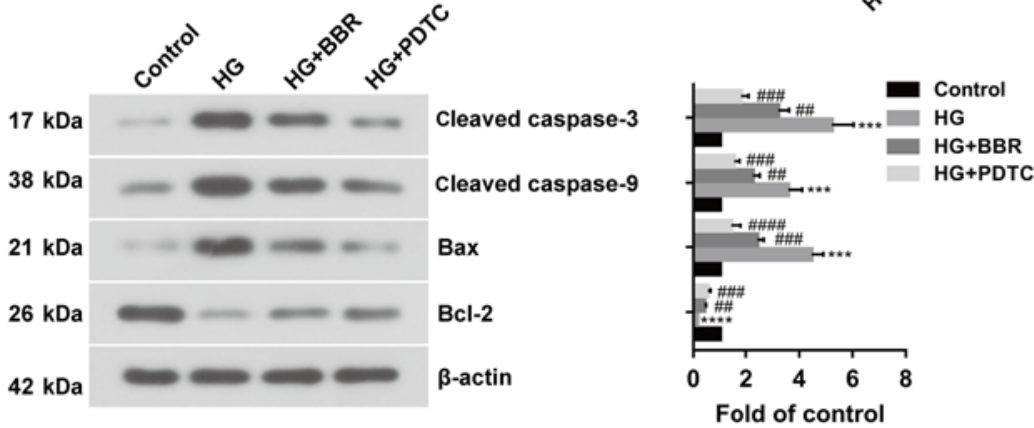
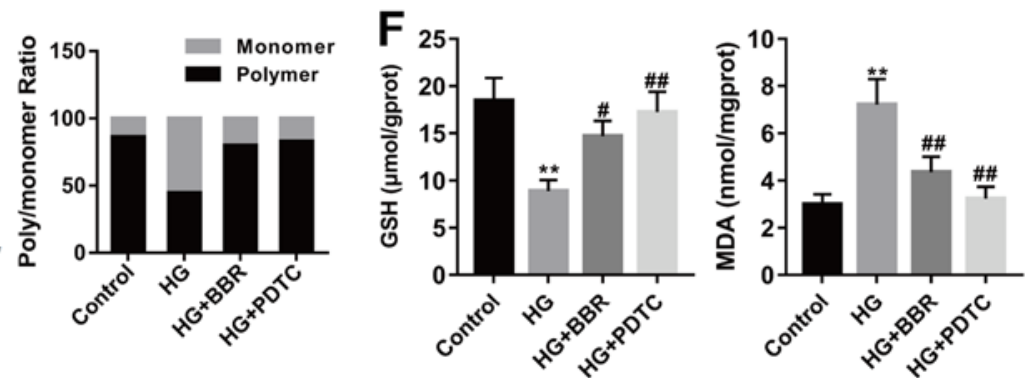

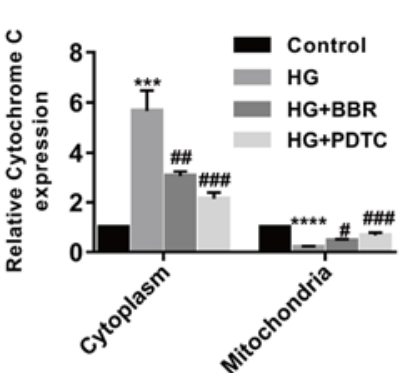

G
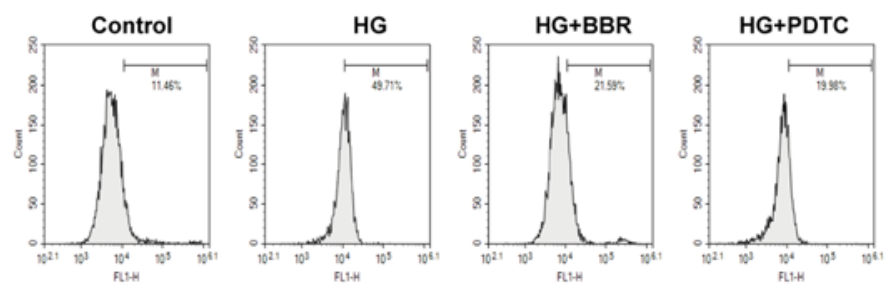
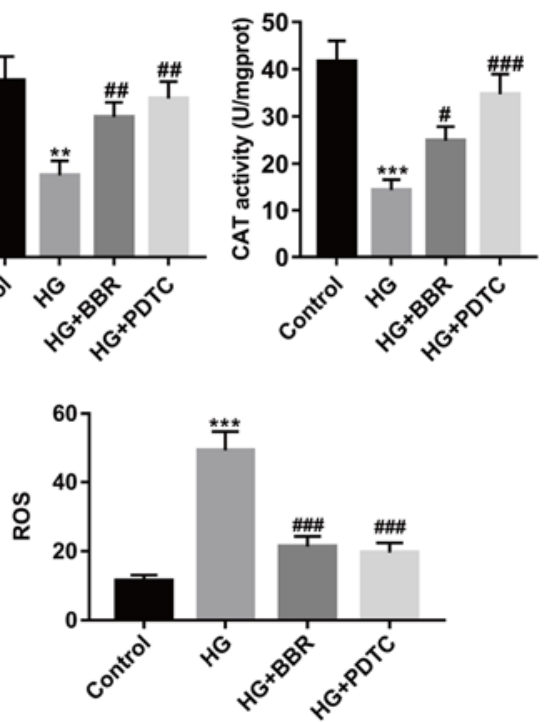

Figure 3. BBR reverses HG-induced effects on cell apoptosis and oxidative stress in Müller cells. PDTC is an NF-kB inhibitor. Müller cells were treated with 5.5 mM glucose; $33.3 \mathrm{mM}$ glucose; $33.3 \mathrm{mM}$ glucose and $20 \mu \mathrm{M}$ BBR; or $33.3 \mathrm{mM}$ glucose and $100 \mu \mathrm{M}$ PDTC. Cell apoptosis was detected using. (A) Annexin V-FITC apoptosis detection and (B) Hoechst staining (scale bar, $50 \mu \mathrm{m}$ ). (C) Protein expression was determined using western blotting. (D) MMP was detected using the JC-1 assay. (E) Cytoplasmic and nuclear expression levels of cytochrome $\mathrm{c}$ were detected using western blotting. (F) GSH, MDA, SOD and CAT contents were measured in treated Müller cells. (G) ROS levels were measured in treated Müller cells. ${ }^{* *} \mathrm{P}<0.01,{ }^{* * * *} \mathrm{P}<0.001$ and ${ }^{* * * * *} \mathrm{P}<0.0001$ vs. the control group. ${ }^{\#} \mathrm{P}<0.05$, ${ }^{\# \#} \mathrm{P}<0.01$ and ${ }^{\# \# \#} \mathrm{P}<0.001$ vs. the HG group. BBR, berberine; HG, high glucose; PDTC, pyrrolidine dithiocarbamate; MMP, mitochondrial membrane potential; GSH, glutathione; MDA, malondialdehyde; SOD, superoxide dismutase; CAT, catalase; COXIV, mitochondrial cytochrome c oxidase subunit IV.

assay, which indicated that BBR or PDTC treatment inhibited $\mathrm{NF}-\kappa \mathrm{B}$ translocation and deactivated the NF- $\mathrm{\kappa B}$ signaling pathway in HG-treated cells (Fig. 4B). The results suggested that BBR decreased cell apoptosis and oxidative stress by deactivating the NF-kB signaling pathway.

\section{Discussion}

BBR is an extract of Rhizoma Coptidis that displays therapeutic activities in several diseases, such as fatty liver, type 2 diabetes and obesity $(10,17)$. DR is diagnosed in patients with 

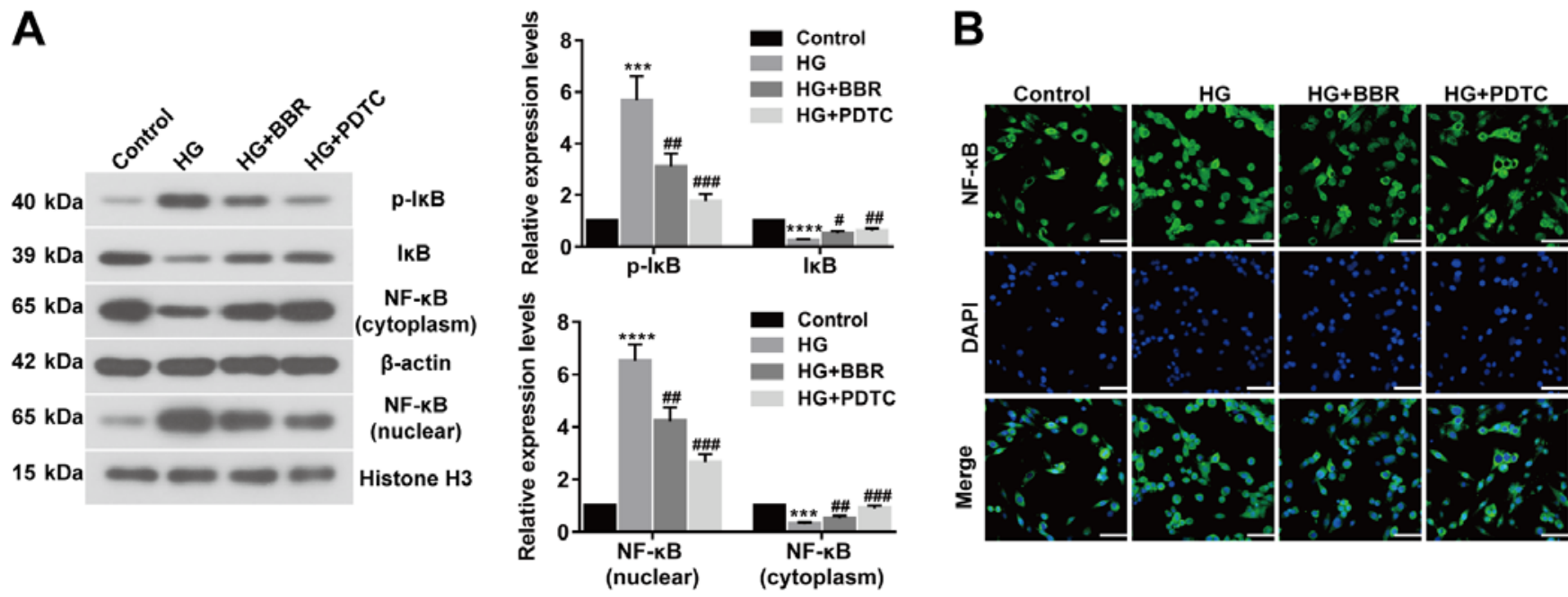

Figure 4. BBR reduces cell apoptosis and oxidative stress by deactivating the NF- $\kappa$ B signaling pathway. (A) Expression levels of p-I $\kappa$ B (Ser32), I $\kappa$ B, NF- $\kappa$ B

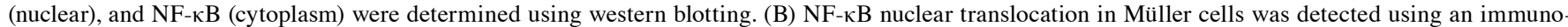
fluorescence assay (scale bar, $50 \mu \mathrm{m}$ ). ${ }^{* * *} \mathrm{P}<0.001$ and ${ }^{* * * *} \mathrm{P}<0.0001$ vs. the control group. ${ }^{\#} \mathrm{P}<0.05,{ }^{\# \#} \mathrm{P}<0.01$ and ${ }^{\# \# \#} \mathrm{P}<0.001$ vs. the $\mathrm{HG}$ group. BBR, berberine; p, phosphorylated; I $\mathrm{B}, \mathrm{NF}-\kappa \mathrm{B}$ inhibitor; $\mathrm{HG}$, high glucose; PDTC, pyrrolidine dithiocarbamate.

diabetes and can lead to blindness (1). Müller cell apoptosis affects the retinal vasculature and neurons, which contributes to retinal complications of diabetes, including DR (18). In the present study, a rat model of DR was established and the results suggested that BBR decreased GCL cell apoptosis, reduced diabetic-induced oxidative stress, and deactivated the $\mathrm{NF}-\kappa \mathrm{B}$ signaling pathway. In vitro experiments indicated that $\mathrm{BBR}$ reversed $\mathrm{HG}$-induced effects on oxidative stress and cell apoptosis in Müller cells by deactivating the NF- $\kappa \mathrm{B}$ signaling pathway. Collectively, in vivo and in vitro results suggested that BBR protected against DR by inhibiting cell apoptosis and reducing oxidative stress via deactivation of the $N F-\kappa B$ signaling pathway.

BBR is a novel organic compound that displays protective effects against multiple diseases (17). For example, BBR displays antioxidant and anti-inflammatory properties, which provide protective effects in neurodegenerative disorders (19). BBR also protects against breast cancer by reducing EGFR and AKT phosphorylation (20). Liang et al (21) reported that BBR ameliorates acute lung injury via the eukaryotic translation initiation factor $2 \alpha$ kinase 3 -mediated nuclear factor erythroid 2-like 2/heme oxygenase 1 signaling axis. BBR participates in the pathogenesis of several diseases by regulating cell apoptosis and oxido-nitrosative stress. BBR can partially suppress the apoptotic cascade and oxido-nitrosative stress, reduce ischemia/reperfusion-induced myocardial apoptosis, and inhibit inflammation and mitochondria-dependent apoptosis in a rat model of diabetes $(19,22,23)$. Furthermore, BBR mitigates HG-induced apoptosis of mouse podocytes in vitro (24). Müller cells form part of the macroglia of the retina, and BBR attenuates apoptosis and injury of HG-induced Müller cells by enhancing autophagy and the AMPK signaling pathway $(11,25)$. BBR can also serve as an inducer of apoptosis, which has been reported in thyroid and hepatocellular carcinoma, as well as colorectal cancer (26-28). Although BBR is a promising agent with anti-carcinogenic activity, the pharmacological properties of BBR also suggest that BBR has a therapeutic effect in diabetes, nonalcoholic fatty liver disease, rheumatoid arthritis, Alzheimer's disease and cardiovascular diseases (29). In vitro and in vivo experiments suggested that BBR may mitigate diabetes-induced retina cell apoptosis and oxidative stress, and have therapeutic effects for DR. However, the mechanism underlying how BBR mitigates DR requires further investigation.

In the present study, BBR alleviated HG-induced cell apoptosis and oxidative stress in Müller cells by deactivating the $\mathrm{NF}-\kappa \mathrm{B}$ signaling pathway. Furthermore, the expression of I $\mathrm{B}$ was decreased and p-IкB was increased in HG-induced Müller cells compared with control cells. BBR or PDTC treatment reversed HG-induced effects. The results suggested that BBR deactivated the NF- $\mathrm{NB}$ signaling pathway by decreasing the phosphorylation of $\mathrm{I} \kappa \mathrm{B}$, resulting in abundant cytoplasmic I $\mathrm{B}$ and inhibition of NF- $\kappa \mathrm{B}$ nuclear translocation. Li et al (28) reported that BBR inhibited the proliferation of HepG2 cells by promoting apoptosis via the NF- $\mathrm{B}$ p 65 signaling pathway. Collectively, the aforementioned results suggest that BBR may serve as a potential therapeutic agent for multiple diseases and different types of cancer; however the underlying mechanisms of action may differ. Similarly, different cell lines may be another reason for distinct interactions with the $N F-\kappa B$ signaling pathway. Therefore, the protective effects of BBR against diseases and different types of cancer requires further investigation.

Oxidative stress is a vital factor for the development of DR (29). Rat models of DR display damaged retinal mitochondria that accelerate apoptosis of retinal cells, and the regulation of oxidative stress protects mitochondrial homeostasis and prevents the development of DR (30). Similarly, mitochondria-induced apoptosis and alterations to oxidative stress were observed in the present study. The oxidation of $\mathrm{H}_{2} \mathrm{O}_{2}$ leads to activation of the NF- $\kappa \mathrm{B}$ signaling pathway by the release of the inhibitory subunit of $\mathrm{I} \kappa \mathrm{B}(31,32)$. In the present study, I $\kappa \mathrm{B}$ expression was decreased in $\mathrm{HG}$-induced Müller cells compared with control cells. BBR or PDTC treatment upregulates $\mathrm{I} \kappa \mathrm{B}$ expression by inhibiting the phosphorylation of I $\kappa$ B. Furthermore, NF- $\kappa$ B nuclear translocation 
was inhibited and the NF- $\mathrm{B}$ signaling pathway was deactivated in the DR group. DNA-binding subunits of NF- $\kappa \mathrm{B}$ containing redox-sensitive cysteine residues inhibit oxidation activity (33). The deactivation of the $\mathrm{NF}-\kappa \mathrm{B}$ signaling pathway reduces DNA-binding to $\mathrm{NF}-\kappa \mathrm{B}$, which inhibits oxidation activity and regulates oxidative stress, resulting in decreased cell apoptosis (33). BBR displayed protective effects against DR-induced cell apoptosis and oxidative stress; however, the mechanisms underlying the effects of BBR in other signaling pathways are not completely understood and require further investigation.

To conclude, BBR protected against DR by inhibiting cell apoptosis and oxidative stress via deactivation of the NF- $\kappa \mathrm{B}$ signaling pathway. The results of the present study may provide a novel therapeutic strategy for DR.

\section{Acknowledgements}

Not applicable.

\section{Funding}

The present study was supported by a grant from the National Natural Science Foundation of China (grant no. 81573746).

\section{Availability of data and materials}

The datasets used and/or analyzed during the current study are available from the corresponding author on reasonable request.

\section{Authors' contributions}

XL designed the study and revised the manuscript. JJZ and $\mathrm{ZL}$ performed the experiments, analyzed the data and wrote the manuscript. HZ, LM, ZM, YZ, JZ, ML, LM and XW performed the experiments and analyzed the data. All authors read and approved the final manuscript.

\section{Ethics approval and consent to participate}

The present study was approved by the Ethics Committee of Xijing Hospital, Air Force Medical University.

\section{Patient consent for publication}

Not applicable.

\section{Competing interests}

The authors declare that they have no competing interests.

\section{References}

1. Cheung N, Mitchell $\mathrm{P}$ and Wong TY: Diabetic retinopathy. Lancet 376: 124-136, 2010.

2. Porojan MD, Cătană A, Popp RA, Dumitrascu DL and Bala C: The role of NOS2A $-954 \mathrm{G} / \mathrm{C}$ and vascular endothelial growth factor $+936 \mathrm{C} / \mathrm{T}$ polymorphisms in type 2 diabetes mellitus and diabetic nonproliferative retinopathy risk management. Ther Clin Risk Manag 11: 1743-1748, 2015.
3. Zhao C, Wang W, Xu D, Li H, Li M and Wang F: Insulin and risk of diabetic retinopathy in patients with type 2 diabetes mellitus: Data from a meta-analysis of seven cohort studies. Diagn Pathol 9: 130, 2014.

4. Toft-Kehler AK, Gurubaran IS, Desler C, Rasmussen LJ, Skytt DM and Kolko M: Oxidative stress-induced dysfunction of muller cells during starvation. Invest Ophthalmol Vis Sci 57: 2721-2728, 2016.

5. He XF, Zhang L, Zhang CH, Zhao CR, Li H, Zhang LF, Tian GF, Guo MF, Dai $Z$ and Sui FG: Berberine alleviates oxidative stress in rats with osteoporosis through receptor activator of $\mathrm{NF}-\mathrm{kB} /$ receptor activator of NF-kB ligand/osteoprotegerin (RANK/RANKL/OPG) pathway. Bosn J Basic Med Sci 17: 295-301, 2017.

6. Kowluru RA, Kowluru A, Veluthakal R, Mohammad G, Syed I, Santos JM and Mishra M: TIAM1-RAC1 signalling axis-mediated activation of NADPH oxidase-2 initiates mitochondrial damage in the development of diabetic retinopathy. Diabetologia 57: 1047-1056, 2014.

7. Sun Y, Yuan X, Zhang F, Han Y, Chang X, Xu X, Li Y and Gao X: Berberine ameliorates fatty acid-induced oxidative stress in human hepatoma cells. Sci Rep 7: 11340, 2017.

8. Sadeghnia HR, Kolangikhah M, Asadpour E, Forouzanfar F and Hosseinzadeh $\mathrm{H}$ : Berberine protects against glutamate-induced oxidative stress and apoptosis in PC12 and N2a cells. Iran J Basic Med Sci 20: 594-603, 2017.

9. Hsu YY, Chen CS, Wu SN, Jong YJ and Lo YC: Berberine activates $\mathrm{Nrf} 2$ nuclear translocation and protects against oxidative damage via a phosphatidylinositol 3-kinase/Akt-dependent mechanism in NSC34 motor neuron-like cells. Eur J Pharm Sci 46: 415-425, 2012.

10. Lan J, Zhao Y, Dong F, Yan Z, Zheng W, Fan J and Sun G: Meta-analysis of the effect and safety of berberine in the treatment of type 2 diabetes mellitus, hyperlipemia and hypertension. J Ethnopharmacol 161: 69-81, 2015.

11. Fu D, Yu JY, Connell AR, Yang S, Hookham MB, McLeese R and Lyons TJ: Beneficial effects of berberine on oxidized LDL-induced cytotoxicity to human retinal muller cells. Invest Ophthalmol Vis Sci 57: 3369-3379, 2016.

12. Tian P, Ge H, Liu H, Kern TS, Du L, Guan L, Su S and Liu P. Leukocytes from diabetic patients kill retinal endothelial cells: Effects of berberine. Mol Vis 19: 2092-2105, 2013.

13. Song D, Song J, Wang C, Li Y and Dunaief JL: Berberine protects against light-induced photoreceptor degeneration in the mouse retina. Exp Eye Res 145: 1-9, 2016.

14. Kronfeld PC: The association for research in vision and ophthalmology. Am J Ophthalmol 76: 305-306, 1973.

15. National Research Council: Guide for the care and use of laboratory animals: Eighth edition. Publication No. 85-23(rev.) 327 pp963-965, 2010.

16. Coughlin BA, Feenstra DJ and Mohr S: Müller cells and diabetic retinopathy. Vision Res 139: 93-100, 2017.

17. Sun Y, Xia M, Yan H, Han Y, Zhang F, Hu Z, Cui A, Ma F, Liu Z, Gong Q, et al: Berberine attenuates hepatic steatosis and enhances energy expenditure in mice by inducing autophagy and fibroblast growth factor 21. Br J Pharmacol 175: 374-387, 2018.

18. Cuenca N, Fernández-Sánchez L, Campello L, Maneu V, De la Villa P, Lax P and Pinilla I: Cellular responses following retinal injuries and therapeutic approaches for neurodegenerative diseases. Prog Retin Eye Res 43: 17-75, 2014.

19. Sadraie S, Kiasalari Z, Razavian M, Azimi S, Sedighnejad L, Afshin-Majd S, Baluchnejadmojarad T and Roghani M: Berberine ameliorates lipopolysaccharide-induced learning and memory deficit in the rat: Insights into underlying molecular mechanisms. Metab Brain Dis 34: 245-255, 2019.

20. Jabbarzadeh Kaboli P, Leong MP, Ismail P and Ling KH: Antitumor effects of berberine against EGFR, ERK1/2, P38 and AKT in MDA-MB231 and MCF-7 breast cancer cells using molecular modelling and in vitro study. Pharmacol Rep 71: 13-23, 2019.

21. Liang Y, Fan C, Yan X, Lu X, Jiang H, Di S, Ma Z,Feng Y,Zhang Z, Feng $\mathrm{P}$, et al: Berberine ameliorates lipopolysaccharide-induced acute lung injury via the PERK-mediated $\mathrm{Nrf} 2 / \mathrm{HO}-1$ signaling axis. Phytother Res 33: 130-148, 2019.

22. Chen K, Li G, Geng F, Zhang Z, Li J, Yang M, Dong L and Gao F: Berberine reduces ischemia/reperfusion-induced myocardial apoptosis via activating AMPK and PI3K-Akt signaling in diabetic rats. Apoptosis 19: 946-957, 2014.

23. Xu L, Zheng X, Wang Y, Fan Q, Zhang M, Li R, Ye J, Wu X, Zhao $\mathrm{W}$ and Zhang Y: Berberine protects acute liver failure in mice through inhibiting inflammation and mitochondriadependent apoptosis. Eur J Pharmacol 819: 161-168, 2018 
24. Jin Y, Liu S, Ma Q, Xiao D and Chen L: Berberine enhances the AMPK activation and autophagy and mitigates high glucose-induced apoptosis of mouse podocytes. Eur J Pharmacol 794: 106-114, 2017.

25. Chen H, Ji Y, Yan X, Su G, Chen L and Xiao J: Berberine attenuates apoptosis in rat retinal Müller cells stimulated with high glucose via enhancing autophagy and the AMPK/mTOR signaling. Biomed Pharmacother 108: 1201-1207, 2018.

26. Li L, Wang X, Sharvan R, Gao J and Qu S: Berberine could inhibit thyroid carcinoma cells by inducing mitochondrial apoptosis G0/G1 cell cycle arrest and suppressing migration via PI3K-AKT and MAPK signaling pathways. Biomed Pharmacother 95: 1225-1231, 2017

27. Dai W, Mu L, Cui Y, Li Y, Chen P, Xie H and Wang X: Berberine promotes apoptosis of colorectal cancer via regulation of the long non-coding RNA (lncRNA) cancer susceptibility candidate 2 (CASC2)/AU-binding factor 1 (AUF1)/B-cell CLL/lymphoma 2 (Bcl-2) axis. Med Sci Monit 25: 730-738, 2019.

28. Li M, Zhang M, Zhang ZL, Liu N, Han XY, Liu QC, Deng WJ and Liao CX: Induction of apoptosis by berberine in hepatocellular carcinoma HepG2 cells via downregulation of NF- $\kappa \mathrm{B}$. Oncol Res 25: 233-239, 2017.
29. Brownlee M: The pathobiology of diabetic complications: A unifying mechanism. Diabetes 54: 1615-1625, 2005.

30. Kowluru RA and Shan Y: Role of oxidative stress in epigenetic modification of MMP-9 promoter in the development of diabetic retinopathy. Graefes Arch Clin Exp Ophthalmol 255: 955-962, 2017.

31. Sies H: Hydrogen peroxide as a central redox signaling molecule in physiological oxidative stress: Oxidative eustress. Redox Biol 11: 613-619, 2017.

32. Schreck R, Rieber P and Baeuerle PA: Reactive oxygen intermediates as apparently widely used messengers in the activation of the NF-kappa B transcription factor and HIV-1. EMBO J 10: 2247-2258, 1991.

33. Toledano MB and Leonard WJ: Modulation of transcription factor NF-kappa B binding activity by oxidation-reduction in vitro. Proc Natl Acad Sci USA 88: 4328-4332, 1991.

(i) (9) This work is licensed under a Creative Commons Attribution-NonCommercial-NoDerivatives 4.0 International (CC BY-NC-ND 4.0) License. 